ISSN: 2456-8686, 5(1), 2021:097-104

https://doi.org/10.26524/cm96

ORIGINAL RESEARCH

Open Access

\title{
Pythagorean Fuzzy Semi Open Sets
}

Ajay D*:1, Annamalai $\mathrm{D}^{2}$ and Joseline Charisma $\mathrm{J}^{3}$

Received: 28 December 2020/ Accepted: 15 March 2021/ Published online: 18 June 2021

(C)Sacred Heart Research Publications 2017

\begin{abstract}
Our main aim is to define the concept of fuzzy semi open sets in Pythagorean fuzzy topological spaces which is one of the weakest forms of open sets. Along with their introduction their characterizations and properties have been investigated with examples. In addition to that the Pythagorean fuzzy continuous functions have also been defined.
\end{abstract}

Key words: Pythagorean Fuzzy Topological Spaces, Pythagorean Fuzzy Open Set, Fuzzy Semi Open, Fuzzy Continuous Function.

AMS classification: 03E72, 54A40.

\section{Introduction}

L.A. Zadeh [1] came up with the idea of fuzzy sets, which is a generalization of the usual set using fuzzy where each element has degree of membership in $[0,1]$. The consequent advancement of fuzzy subsets was the intuitionistic fuzzy set published by Atanassov [2] which has elements having membership and non-membership degree. Chang [3] defined fuzzy topological space and fundamental results such as continuity, open and closed sets. Following this, Lowen [4] defined fuzzy topological space in another form. Coker [5] developed the concept of an intuitionistic fuzzy topological spaces with some properties. The concept of Pythagorean fuzzy subset was presented by Yager [6, 7]. Pythagorean fuzzy topological spaces was introduced by Olgun [8] by taking the lead from Chang. In [9, 10, 11, 12, 13, 14] the concepts like connectedness, points, continuity and nano topology have been defined for Pythagorean topological spaces. The concept of semi open set and continuous functions of Pythagorean topological spaces are defined and their properties are to be examined in section 2 and concluded in section 3.

\footnotetext{
${ }^{1,2,3}$ Department of Mathematics, Sacred Heart College (Autonomous), Tirupattur - 635 601, Tamil Nadu, India Email: *,1 dajaypravin@gmail.com, ${ }^{2}$ annamalaisiva7657@gmail.com, ${ }^{3}$ joecharish@gmail.com
} 


\section{Semi open sets and continuous functions in Pythagorean Fuzzy Topological Spaces}

Definition 2.1 Let $\mathfrak{A}$ be a Pythagorean fuzzy set (PFS) of Pythagorean fuzzy topological space (PFTS) $(W, \tau)$, then $\mathfrak{A}$ is said to be Pythagorean fuzzy semi open (PFSO) set of $W$ if there exists a Pythagorean fuzzy open (PFO) set $\mathfrak{B} \in \tau$ such that $\mathfrak{B} \subseteq \mathfrak{A} \subseteq \operatorname{cl}(\mathfrak{B})$, Pythagorean fuzzy semi closed (PFSC) set of $W$ if there exists a Pythagorean fuzzy closed (PFC) set $\mathfrak{B} \in \tau$ such that $\operatorname{int}(\mathfrak{B}) \subseteq \mathfrak{A} \subseteq \mathfrak{B}$.

In other words, let $\mathfrak{A}$ be a Pythagorean fuzzy set (PFS) of Pythagorean fuzzy topological space (PFTS) $(W, \tau)$, then $\mathfrak{A}$ is said to be Pythagorean fuzzy semi open (PFSO) set of $W$ if $\mathfrak{A} \subseteq \operatorname{cl}(\operatorname{int}(\mathfrak{A}))$.

Remark 2.2 It is obvious that every PFO (PFC) set in a PFTS $(W, \tau)$ is PFSO (PFSC) but the converse is not true.

Example 2.3 Let $W=\{a, b, c\}$

$\mathfrak{A}_{1}=\{(a, 0.5,0.8),(b, 0.4,0.6),(c, 0.6,0.3)\}$

$\mathfrak{A}_{2}=\{(a, 0.6,0.9),(b, 0.5,0.3),(c, 0.1,0.9)\}$

$\mathfrak{A}_{3}=\{(a, 0.6,0.8),(b, 0.5,0.3),(c, 0.6,0.3)\}$

$\mathfrak{A}_{4}=\{(a, 0.5,0.3),(b, 0.5,0.3),(c, 0.1,0.3)\}$

$\tau=\left\{0_{\sim}, 1_{\sim}, \mathfrak{A}_{1}, \mathfrak{A}_{2}, \mathfrak{A}_{3}, \mathfrak{A}_{4}\right\}$ be a Pythagorean fuzzy topology on W. Let

$\mathfrak{A}=\{(a, 0.6,0.8),(b, 0.9,0.3),(c, 0.6,0.4)\}, \operatorname{int}(\mathfrak{A})=\{(a, 0.6,0.8),(b, 0.5,0.3)$,

$(c, 0.6,0.3)\}, \operatorname{int}(\mathfrak{A})=\mathfrak{A}_{3}$.

Let the Pythagorean fuzzy semi closed sets be,

$\mathfrak{A}_{1}^{\prime}=\{(a, 0.8,0.5),(b, 0.6,0.4),(c, 0.3,0.6)\}$

$\mathfrak{A}_{2}^{\prime}=\{(a, 0.9,0.6),(b, 0.3,0.5),(c, 0.9,0.1)\}$

$\mathfrak{A}_{3}^{\prime}=\{(a, 0.8,0.6),(b, 0.3,0.5),(c, 0.3,0.6)\}$

$\mathfrak{A}_{4}^{\prime}=\{(a, 0.9,0.5),(b, 0.6,0.4),(c, 0.9,0.1)\}$

$\operatorname{cl}(\mathfrak{A})=\{(a, 0.5,0.9),(b, 0.4,0.6),(c, 0.1,0.9)\}$

$\operatorname{cl}(\mathfrak{A})=1_{\sim}, \mathfrak{A} \subseteq \operatorname{cl}(\operatorname{int}(\mathfrak{A})), \mathfrak{A} \subseteq \operatorname{cl}\left(\mathfrak{A}_{3}\right), \mathfrak{A} \subseteq 1_{\sim} . \therefore \mathfrak{A}$ is a Pythagorean fuzzy semi open set.

Theorem 2.4 Let $(W, \tau)$ be a PFTS. Let $\mathfrak{A}$ and $\mathfrak{B}$ be any two Pythagorean fuzzy subsets of $W$ and int $\mathfrak{A} \subseteq \mathfrak{B} \subseteq \operatorname{cl}(\mathfrak{A})$. If $\mathfrak{A}$ is a PFSO set then $\mathfrak{B}$ is PFSO. 
Proof: Let $\mathfrak{A}$ and $\mathfrak{B}$ be a Pythagorean fuzzy subsets of $W$ and $\operatorname{int}(\mathfrak{B}) \subseteq \mathfrak{B} \subseteq \operatorname{cl}(\mathfrak{A})$. Let $\mathfrak{A}$ be PFSO set. By definition, there exists a PFO $\mathfrak{O}$ such that $\mathfrak{O} \subseteq \mathfrak{A} \subseteq \operatorname{cl}(\mathfrak{O})$, it follows that $\mathfrak{O} \subseteq \operatorname{int}(\mathfrak{A}) \subseteq \mathfrak{A} \subseteq \operatorname{cl}(\mathfrak{A}) \subseteq \operatorname{cl}(\mathfrak{O})$ and hence $\mathfrak{O} \subseteq \mathfrak{B} \subseteq \operatorname{cl}(\mathfrak{O})$. Thus $\mathfrak{B}$ is a PFSO set.

Theorem 2.5 Let $(W, \tau)$ be a PFTS. Then a Pythagorean fuzzy subset $\mathfrak{A}$ of a PFTS $(W, \tau)$ is PFSO if and only if $\mathfrak{A} \subseteq \operatorname{cl}(\operatorname{int}(\mathfrak{A}))$.

Proof: Let $\mathfrak{A} \subseteq \operatorname{cl}(\operatorname{int}(\mathfrak{A}))$. Then for $\mathfrak{O}=\operatorname{int}(\mathfrak{A})$, we have $\operatorname{int}(\mathfrak{A}) \subseteq \mathfrak{A}$. Therefore $\operatorname{int}(\mathfrak{A}) \subseteq \mathfrak{A} \subseteq \operatorname{cl}(\operatorname{int}(\mathfrak{A}))$. Conversely, let $\mathfrak{A}$ be PFSO. By definition, there exists a PFO $\mathfrak{O}$ such that $\mathfrak{O} \subseteq \mathfrak{A} \subseteq \operatorname{cl}(\mathfrak{O})$. But $\mathfrak{O} \subseteq \operatorname{int}(\mathfrak{A})$, thus $\operatorname{cl}(\mathfrak{O}) \subseteq \operatorname{cl}(\operatorname{int}(\mathfrak{A}))$. Hence $\mathfrak{A} \subseteq \operatorname{cl}(\mathfrak{O}) \subseteq \operatorname{cl}(\operatorname{int}(\mathfrak{A}))$.

Theorem 2.6 Let $(W, \tau)$ be a PFTS. Then the following are equivalent:

(i) $\mathfrak{A}$ is PFSC set

(ii) $\overline{\mathfrak{A}}$ is PFSO set

(iii) int $\operatorname{cl}(\mathfrak{A}) \leq \mathfrak{A}$

(iv) $\operatorname{cl} \operatorname{int}(\overline{\mathfrak{A}}) \geq \overline{\mathfrak{A}}$

Proof: (i) $\Longrightarrow$ (ii) By definition there exist a closed set $\mathfrak{B}$ in $(W, \tau)$ such that $\operatorname{int}(\mathfrak{B}) \leq \mathfrak{A} \leq \mathfrak{B}$, this implies $\overline{\operatorname{int}(\mathfrak{B})} \geq \overline{\mathfrak{A}} \geq \overline{\mathfrak{B}}$. By $\operatorname{cl} \overline{(\mathfrak{A})}=\overline{\operatorname{int}(\mathfrak{A})}$, we get $\overline{(\mathfrak{B})} \leq \overline{\mathfrak{A}}$ $\leq \mathrm{cl}(\overline{\mathfrak{B}})$ where $\overline{(\mathfrak{B})}$ is a PFO set in $(W, \tau)$. Therefore, $\overline{\mathfrak{A}}$ is PFSO in $(W, \tau)$.

(ii) $\Longrightarrow$ (i) Given $\overline{\mathfrak{A}}$ is PFSO set in $(W, \tau)$, that is $\overline{\mathfrak{B}} \subseteq \overline{\mathfrak{A}} \subseteq \operatorname{cl}(\overline{\mathfrak{B}})$ where $\overline{\mathfrak{B}}$ is PFO set in $(W, \tau)$. By $\operatorname{cl} \overline{(\mathfrak{A})}=\overline{\operatorname{int}(\mathfrak{A})}$, this implies $\overline{\operatorname{int}(\mathfrak{B})} \geq \overline{\mathfrak{A}} \geq \overline{\mathfrak{B}}$. Then we get $\operatorname{int}(\mathfrak{B}) \leq \mathfrak{A} \leq \mathfrak{B}$. That is $\mathfrak{B}$ is closed set in $(W, \tau)$. Therefore $\mathfrak{A}$ is PFSO set.

(i) $\Longrightarrow$ (iii) By definition there exists a PFC set $\mathfrak{B}$ in $(W, \tau)$ such that $\operatorname{int}(\mathfrak{B}) \leq \mathfrak{A} \leq \mathfrak{B}$ and $\mathfrak{A} \leq \operatorname{cl}(\mathfrak{A})$ we get, $\operatorname{int}(\mathfrak{B}) \leq \mathfrak{A} \leq \operatorname{cl}(\mathfrak{A}) \leq \mathfrak{B}$. Since $\operatorname{int}(\mathfrak{B})$ is the largest open set contained in $\mathfrak{B}$, we have that $\operatorname{int} \operatorname{cl}(\mathfrak{A}) \leq \operatorname{int}(\mathfrak{B}) \leq \mathfrak{A}$ i.e, $\operatorname{int} \operatorname{cl}(\mathfrak{A}) \leq \mathfrak{A}$.

(iii) $\Longrightarrow($ i) This follows by taking $\mathfrak{B}=\operatorname{cl}(\mathfrak{A})$ i.e, $\operatorname{int}(\mathfrak{A})=\mathfrak{B}$. Also we know that $\mathfrak{A} \leq \operatorname{cl}(\mathfrak{A})=\mathfrak{B}$ and hence $\operatorname{int}(\mathfrak{B}) \leq \mathfrak{A} \leq \mathfrak{B}$ as $\mathfrak{B}=\operatorname{cl}(\mathfrak{A})$ is closed set. Therefore $\mathfrak{A}$ is PFSC.

(ii) $\Longrightarrow$ (iv) Given $\overline{\mathfrak{A}}$ is PFSO set in $(W, \tau)$. That is $\overline{\mathfrak{B}} \subseteq \overline{\mathfrak{A}} \subseteq \operatorname{cl}(\overline{\mathfrak{B}})$. Then we have, $\overline{\operatorname{int}(\mathfrak{B})} \geq \overline{\mathfrak{A}}$. Therefore, $\operatorname{cl} \operatorname{int}(\overline{\mathfrak{A}}) \geq \overline{\mathfrak{A}}$. 
Theorem 2.7 Union of a finite number of PFSO sets is a PFSO set and intersection of a finite number of PFSC sets is a PFSC set.

Proof: Let $\mathfrak{A}_{1}, \mathfrak{A}_{2}, \mathfrak{A}_{3}, \ldots, \mathfrak{A}_{n}$ be PFSO sets of $(W, \tau)$ then there exist PFO sets $\mathfrak{B}_{1}$, $\mathfrak{B}_{2}, \mathfrak{B}_{3}, \ldots, \mathfrak{B}_{n}$ of $(\mathrm{W}, \tau)$ such that $\mathfrak{B}_{i} \subseteq \mathfrak{A}_{i} \subseteq \operatorname{cl}\left(\mathfrak{B}_{i}\right), \mathrm{i}=1,2, \ldots, \mathrm{n}$. We claim that union of PFSO is a PFSO set.

$\Rightarrow \bigcup \mathfrak{B}_{i} \subseteq \bigcup \mathfrak{A}_{i} \subseteq \bigcup \mathrm{cl}\left(\mathfrak{B}_{i}\right) \Rightarrow \bigcup \mathfrak{B}_{i} \subseteq \bigcup \mathfrak{A}_{i} \subseteq \mathrm{cl}\left(\bigcup \mathfrak{B}_{i}\right)$

Also $\bigcup \mathfrak{B}_{i} \in \tau$, Hence $\bigcup \mathfrak{A}_{i}$ is PFSO.

Conversely, let $\mathfrak{A}_{1}, \mathfrak{A}_{2}, \mathfrak{A}_{3}, \ldots, \mathfrak{A}_{n}$ be PFSC sets of $(W, \tau)$. Then their exist PFC sets $\mathfrak{B}_{1}, \mathfrak{B}_{2}, \mathfrak{B}_{3}, \ldots, \mathfrak{B}_{n}$ of $(W, \tau)$. Now we claim that intersection of a finite number of PFSC sets is a PFSC set. Let $\mathfrak{A}$ be a PFSC set, that is $\operatorname{int}(\mathfrak{B}) \supseteq \mathfrak{A} \supseteq \mathfrak{B}$. $\Rightarrow \bigcap \operatorname{int}\left(\mathfrak{B}_{i}\right) \supseteq \bigcap \mathfrak{A}_{i} \supseteq \bigcap \mathfrak{B}_{i} \Rightarrow \operatorname{int}\left(\bigcap \mathfrak{B}_{i}\right) \supseteq \bigcap \mathfrak{A}_{i} \supseteq \bigcap \mathfrak{B}_{i}$. Also $\bigcap \mathfrak{B}_{i} \in \tau$, hence $\cap \mathfrak{A}_{i}$ is PFSC.

Proposition 2.8 Let $(W, \tau)$ be a PFTS and let $\mathfrak{B}=\left\{\left\langle w, \eta_{\beta}(w), \gamma_{\beta}(w)\right\rangle: w \in W\right\}$ be PFSO sets in $W$, then $\eta_{\beta}=\left\{\left\langle w, \eta_{\beta},(w)\right\rangle: w \in W\right\}$ is PFSO in $\left(W, \tau_{1}\right)$ and $\gamma_{\beta}=\left\{\left\langle w, \gamma_{\beta},(w)\right\rangle: w \in W\right\}$ is PFSC in $\left(W, \tau_{2}\right)$, where $\tau_{1}=\left\{\eta_{G}: G \in \tau\right\}$ and $\tau_{2}=\left\{1-\gamma_{G}: G \in \tau\right\}$ are FTS on $W$ in Chang's sense.

Proof: Given $\mathfrak{B}$ is PFSO, then there exists PFO set $\mathfrak{A}$ such that $\mathfrak{A} \subseteq \mathfrak{B} \subseteq \operatorname{cl}(\mathfrak{A})$. Let $\mathfrak{A}=\left\langle w, \eta_{\mathfrak{A}}, \gamma_{\mathfrak{A}}\right\rangle$ and suppose that the family of PFC sets containing $\mathfrak{A}$ are indexed by $\left\{\left\langle w, \gamma_{\mathfrak{G}_{\mathfrak{i}}}, \eta_{\mathfrak{G}_{\mathfrak{i}}}\right\rangle: i \in \mathfrak{J}\right\}$. then we have, $\operatorname{cl}(\mathfrak{A})=\left\langle w, \wedge \gamma_{\mathfrak{G}_{\mathfrak{i}}}, \vee \eta_{\mathfrak{G}_{\mathfrak{i}}}\right\rangle$ and $\eta_{\mathfrak{A}} \leq \gamma_{\mathfrak{G}_{\mathfrak{i}}}, \gamma_{\mathfrak{A}} \geq \eta_{\mathfrak{G}_{\mathfrak{i}}}$ for each $i \in \mathfrak{J}$. By definition of PFSO sets we get,

$\left\langle w, \eta_{\mathfrak{A}}, \gamma_{\mathfrak{A}}\right\rangle \leq\left\langle w, \eta_{\mathfrak{B}}, \gamma_{\mathfrak{B}}\right\rangle \leq\left\langle w, \gamma_{G_{i}}, \eta_{G_{i}}\right\rangle$, each $i \in \mathfrak{J}$

$\eta_{\mathfrak{A}} \leq \gamma_{\mathfrak{G}_{\mathfrak{i}}} \leq \gamma_{\mathfrak{G}_{\mathfrak{i}}}$ and $\gamma_{\mathfrak{A}} \geq \gamma_{\mathfrak{A}} \geq \eta_{\mathfrak{G}_{\mathfrak{i}}}$, each $i \in \mathfrak{J}$

$\eta_{\mathfrak{A}} \leq \gamma_{\mathfrak{G}_{\mathfrak{i}}} \leq\left(1-\eta_{\mathfrak{G}_{\mathfrak{i}}}\right)$ and $\left(1-\gamma_{\mathfrak{G}_{\mathfrak{i}}}\right) \leq \gamma_{\mathfrak{B}} \leq \gamma_{\mathfrak{A}}$, each $i \in \mathfrak{J}$

$\eta_{\mathfrak{A}} \leq \gamma_{\mathfrak{G}_{\mathfrak{i}}} \leq \operatorname{cl}\left(\eta_{\mathfrak{G}_{\mathfrak{i}}}\right)$ and $\operatorname{int}\left(\gamma_{\mathfrak{A}}\right) \leq \gamma_{\mathfrak{B}} \leq \gamma_{\mathfrak{A}}$

i.e, $\eta_{\mathfrak{B}}=\left\{\left\langle w, \eta_{\mathfrak{B}},(w)\right\rangle: w \in W\right\}$ is PFSO in $\left(W, \tau_{1}\right)$ and $\gamma_{\mathfrak{B}}=\left\{\left\langle w, \gamma_{\mathfrak{B}},(w)\right\rangle: w \in W\right\}$ is PFSC in $\left(W, \tau_{2}\right)$.

Example 2.9 Any FTS $\left(W, \tau_{0}\right)$ obviously a PFTS in the form $\tau=\left\{\mathfrak{A}: \eta_{\mathfrak{A}} \in \tau_{0}\right\}$ whenever we identify a fuzzy set in $W$ whose membership function in $\eta_{\mathfrak{A}}$ with its counter part $\mathfrak{A}=\left\langle w, \eta_{\mathfrak{A}},\left(1-\eta_{\mathfrak{A}}\right)\right\rangle$. If $\eta_{\beta}$ is fuzzy semi open in $\left(W, \tau_{0}\right)$ then $\mathfrak{B}=$ $\left\langle w, \eta_{\mathfrak{B}},\left(1-\eta_{\mathfrak{B}}\right)\right\rangle$ is PFSO in $\left(W, \tau_{0}\right)$

Since $\eta_{\mathfrak{B}}$ is semi open in $\left(W, \tau_{0}\right)$, there exists a open set in $\left(W, \tau_{0}\right)$ such that $\eta_{\mathfrak{A}} \leq$ $\gamma_{\mathfrak{G}_{\mathfrak{i}}} \leq \operatorname{cl}\left(\eta_{\mathfrak{G}_{\mathfrak{i}}}\right)$, i.e, $\left(1-\eta_{\mathfrak{A}}\right) \geq\left(1-\eta_{\mathfrak{B}}\right) \geq 1-\operatorname{cl}\left(\eta_{\mathfrak{A}}\right)=\operatorname{int}\left(1-\eta_{\mathfrak{A}}\right)$, i.e, $\operatorname{int}\left(1-\eta_{\mathfrak{A}}\right) \leq$ $1-\eta_{\mathfrak{B}} \leq 1-\eta_{\mathfrak{A}}$. Therefore $\left(1-\eta_{\mathfrak{B}}\right)$ is PFSC in $\left(W, \tau_{0}\right)$. Thus we conclude that $\mathfrak{A}=\left\langle w, \eta_{\mathfrak{A}}, 1-\eta_{\mathfrak{A}}\right\rangle \leq \mathfrak{B}=\left\langle w, \eta_{\mathfrak{B}}, 1-\eta_{\mathfrak{B}}\right\rangle \leq \operatorname{cl}(\mathfrak{A})$. Hence $\mathfrak{B}$ is PFSO in $(W, \tau)$. 
Definition 2.10 Let $(W, \tau)$ and $(X, \phi)$ be two PFTS's and $f: W \rightarrow X$ a function. Then $f$ is said to be Pythagorean fuzzy semi continuous (PFSCn) iff the pre image of each PFO set in $\phi$ is a PFSO in $\tau$.

Remark 2.11 Every Pythagorean fuzzy continuous function is a Pythagorean fuzzy semi continuous but the converse is not true.

Example 2.12 Let $W=[a, b]$. Consider the PF sets on $W$ are $\mathfrak{A}=\{(a, 0.8,0.7)$, $(b, 0.8,0.8)\} \mathfrak{B}=\{(a, 0.8,0.7),(b, 0.8,0.2)\}$. Then $\tau=\left\{0_{\sim}, 1_{\sim}, \mathfrak{A}\right\}$ is a PFT on $W$. Consider a function $f:(W, \tau) \rightarrow(W, \tau)$ defined by $f(a)=f(b)=a$. Since $f^{-1}=$ $\mathfrak{B} \notin(W, \tau), f$ is PFSCn but it is not PF continuous.

Definition 2.13 Let $(W, \tau)$ and $(W, \phi)$ be two PFTS and $f: W \rightarrow X$ a function. Then $f$ is said to be PFSO mapping if the image of each PFO set $\tau$ is an PFSO set in $\phi$. It is obvious that every PFO mapping is PF mapping.

Example 2.14 Let $(W, \tau)$ and $(X, \phi)$ be two FTS's in the sense of Chang.

(a) If $f: W \rightarrow X$ is PFSCn in the usual sense, then in this case, $f$ is PFS continuous. Here we consider the PFS's on $W$ and $X$ are as follows. $\tau=\left\{\left\langle w, \eta_{\mathfrak{G}}, 1-\eta_{\mathfrak{G}}\right\rangle, \eta_{\mathfrak{G}} \in \tau_{0}\right\}$ and $\phi=\left\{\left\langle x, \lambda_{\mathfrak{H}}, 1-\lambda_{\mathfrak{H}}\right\rangle: \lambda_{\mathfrak{H}} \in \phi_{0}\right\}$. In this case we have for each $\left\langle x, \lambda_{\mathfrak{H}}, 1-\lambda_{\mathfrak{H}}\right\rangle \in \phi, \lambda_{\mathfrak{H}} \in \phi_{0}$ $f^{-1}\left(\left\langle x, \lambda_{\mathfrak{H}}, 1-\lambda_{\mathfrak{H}}\right\rangle\right)=\left\langle w, f^{-1}\left(\lambda_{\mathfrak{H}}\right), f^{-1}\left(1-\lambda_{\mathfrak{H}}\right)\right\rangle$ is semi open set in $\tau$. As $\lambda_{\mathfrak{H}} \in$ $\phi_{0}$ and $f$ is fuzzy semi open, therefore $f^{-1}\left(\lambda_{\mathfrak{H}}\right)$ is PFSO in $\left(W, \tau_{0}\right)$ and $1-f^{-1}\left(\lambda_{\mathfrak{H}}\right)$ is $\operatorname{PFSC}$ in $(W, \tau)$.

(b) Let $f: W \rightarrow X$ be a PFSO function in the usual sense. Then $f$ is also PFSO function in the sense of above definition.

Theorem 2.15 A function $f:(W, \tau) \rightarrow(X, \tau)$ is PFSCn iff $f^{-1}(\mathfrak{B})$ is a PFSC set in $W$, for each $\mathrm{PFC}$ set $\mathfrak{B}$ in $X$.

Proof: Let $f$ is PFSCn and also let $\mathfrak{B}$ be any PF closed in $X$, then $\overline{\mathfrak{B}}$ is a PFO set in $X$. By definition of PFSCn function $f^{-1}(\overline{\mathfrak{B}})$ is PFSO in $(W, \tau)$.

$\overline{f^{-1}(\mathfrak{B})}$ is PFSO in $(W, \tau)$. i.e, $f^{-1}(\mathfrak{B})$ is $\operatorname{PFSC}$ in $(W, \tau)$.

Conversely, let $f^{-1}(\mathfrak{B})$ is PFSC in $(W, \tau) . \Rightarrow \overline{f^{-1}(\mathfrak{B})}$ is PFSO in $(W, \tau) . \Rightarrow f^{-1}(\overline{\mathfrak{B}})$ is PFSO in $(W, \tau)$ by definition of PFSCn. $\overline{\mathfrak{B}}$ is a PFO set in $X \Rightarrow \mathfrak{B}$ be any $\mathrm{PF}$ closed set in $X$. Therefore $f$ is PFSCn. 
Theorem 2.16 If $f$ is PFSCn function and $g$ is PF continuous then $g \circ f$ is PFSCn function.

Proof: Let $f:(W, \tau) \rightarrow(X, \phi)$ and $g:(X, \phi) \rightarrow(Y, \beta)$ be two PFSCn functions. For $\eta \in \beta$, let $(g \circ f)^{-1}(\eta)=f^{-1}(\eta \circ g)=f^{-1}\left(g^{-1}(\eta)\right)$. Since $g$ is Pythagorean fuzzy continuous, $g^{-1}(\eta) \in \phi . \quad g^{-1}(\eta)$ be a PFS open set in $(X, \phi)$ and since $f$ is Pythagorean fuzzy continuous,$f^{-1}\left(g^{-1}(\eta)\right)$ is Pythagorean fuzzy semi open in $\tau$. Thus $f^{-1}\left(g^{-1}(\eta)\right) \in \tau . \therefore g \circ f$ is PFSCn function.

Definition 2.17 Let $(W, \tau)$ be a Pythagorean fuzzy topological space.

(i) $W$ is said to be Pythagorean fuzzy disconnected, if there exist Pythagorean fuzzy open sets $\mathfrak{A} \neq 1_{w}$ and $\mathfrak{A} \neq 0_{w}$ such that $\mathfrak{A} \cup \mathfrak{B}=1_{w}$ and $\mathfrak{A} \cap \mathfrak{B}=0_{w}$.

(ii) $W$ is said to be Pythagorean fuzzy connected, if $W$ is not a Pythagorean fuzzy disconnected.

Definition 2.18 Let $(W, \tau)$ is a PFTS.

(i) $W$ is said to be Pythagorean fuzzy $C_{5}$ - disconnected if there exists a Pythagorean fuzzy open and fuzzy closed set $\mathfrak{M}$ such that $\mathfrak{M} \neq 1_{w}$ and $\mathfrak{M} \neq 0_{w}$.

(ii) $W$ is said to be Pythagorean fuzzy $C_{5}$ - connected if it is not Pythagorean fuzzy $C_{5}$ - disconnected.

Example 2.19 Let $W=\{a, b\}$ and define the Pythagorean fuzzy subsets $\mathfrak{A}, \mathfrak{B}, \mathfrak{C}, \mathfrak{D}$ as follows, $\mathfrak{A}=\{(a, 0.4,0.3),(b, 0.2,0.7)\}, \mathfrak{B}=\{(a, 0.3,0.4),(b, 0.7,0.2)\}$, $\mathfrak{C}=\{(a, 0.3,0.4),(b, 0.2,0.7)\}, \mathfrak{D}=\{(a, 0.4,0.3),(b, 0.7,0.2)\}$.

Then $\tau=\left\{0_{\sim}, 1_{\sim}, \mathfrak{A}, \mathfrak{B}, \mathfrak{C}, \mathfrak{D}\right\}$ is called a Pythagorean fuzzy topology on $W$ and $(W, \tau)$ is a Pythagorean fuzzy $C_{5}$ - disconnected, since $\mathfrak{A}$ is a non zero Pythagorean fuzzy open and Pythagorean fuzzy closed set in $W$.

Definition 2.20 A Pythagorean fuzzy topological space $(W, \tau)$ is said to be Pythagorean fuzzy semi $C_{5}$ - connected if the only Pythagorean fuzzy sets which are both PFSO and PFSC are $0_{\sim}$ and $1_{\sim} .(W, \tau)$ is said to be PF semi $C_{5}$-disconnected if it is not PF semi $C_{5}$ - connected.

Theorem 2.21 Let $f:(W, \tau) \rightarrow(X, \phi)$ be a PFSCn surjection. If $(W, \tau)$ is fuzzy (IT) 
ISSN: 2456-8686, 5(1), 2021:097-104

https://doi.org/10.26524/cm96

semi $C_{5}$ - connected, then $(X, \phi)$ is fuzzy $C_{5}$ - connected.

Proof: We shall prove by contradiction, suppose that $(X, \phi)$ is fuzzy $C_{5}$ - disconnected. Then there exists a PFO and PFC set $\mathfrak{M}$ such that $\mathfrak{M} \neq 1_{\sim}$ and $\mathfrak{M} \neq 0_{\sim}$. Since $f$ is PFSCn, $f^{-1}(\mathfrak{M})$ is both a PFSO and PFSC set. The equalities $f^{-1}(\mathfrak{M})=1 \sim$ or $f^{-1}(\mathfrak{M})=0_{\sim}$ cannot hold. (otherwise we get $\mathfrak{M}=f\left(f^{-1}(\mathfrak{M})\right)=f\left(1_{\sim}\right)=1_{\sim}$ and $\left.\mathfrak{M}=f\left(f^{-1}(\mathfrak{M})\right)=f\left(0_{\sim}\right)=0_{\sim}\right)$. Hence $(X, \phi)$ is PF $C_{5}$ - connected.

\section{Conclusions}

In this paper, the semi open sets for Pythagorean fuzzy topological spaces have been defined. Along with it, their properties and characterizations have been investigated with examples. This study can be extended to various forms of fuzzy open and closed sets in Pythagorean fuzzy topological spaces and their properties can be examined.

\section{References}

[1] Zadeh L A, Fuzzy Sets. Inform. and Control, 8, 338 - 353, (1965).

[2] Atanassov K, Intuitionistic Fuzzy Sets. Fuzzy Sets and Systems, 20, 87 - 96, (1986).

[3] Chang C L, Fuzzy topological spaces. Journal of mathematical Analysis and Applications, 24(1), 182-190, (1968).

[4] Lowen R, Fuzzy topological spaces and fuzzy compactness, Journal of Mathematical analysis and applications, 56(3), 621-633, (1976).

[5] Coker D, An introduction to intuitionistic fuzzy topological spaces, Fuzzy sets and systems, 88(1), 81-89, (1997).

[6] Yager R R, Abbasov A M, Pythagorean membership grades, complex numbers, and decision making, International Journal of Intelligent Systems, 28(5), 436-452, (2013).

[7] Yager R. R., Pythagorean membership grades in multicriteria decision making, IEEE Trans., Fuzzy Syst., 22(4), 958-965, (2014).

[8] Olgun M, Ünver M, Yardimci S, Pythagorean fuzzy topological spaces, Complex and Intelligent Systems, 5(2), 177-183, (2019). 
ISSN: 2456-8686, 5(1), 2021:097-104

https://doi.org/10.26524/cm96

[9] Es A H, Connectedness in Pythagorean fuzzy topological spaces, International Journal of Mathematics Trends and Technology, 65, 110-116, (2019).

[10] Olgun M, Ünver M, Yardimci S, Pythagorean fuzzy points and applications in pattern recognition and Pythagorean fuzzy topologies, Soft Computing, 25(7), 5225-5232, (2021).

[11] Ajay D, Charisma J J, Pythagorean fuzzy $\alpha$-continuity, Journal of Computational Mathematica, 4(2), 10-15, (2020).

[12] Ajay D, Charisma J J, Pythagorean Nano Topological Space, International journal of Recent Technology and Engineering, 8(5), 3415-3419, (2020).

[13] Ajay D, Charisma J J, Pythagorean Nano Continuity, Advances in Mathematics: Scientific Journal, 9(8), 6291-6298, (2020).

[14] Ajay D, Charisma J J, On weak forms of Pythagorean nano open sets, Advances in Mathematics: Scientific Journal, 9(8), 5953-5963, (2020). 